Comment on: "Overestimation of glomerular filtration rate calculated from serum creatinine as compared with cystatin $C$ in patients with subclinical hypercortisolism: Hyogo Adrenal Metabolic Registry" by Naka et al.

\section{Dear Sir;}

In their important cross-sectional study among patients with overt Cushing's syndrome, possible autonomous cortisol secretion and non-functioning adenomas Naka et al. [1] recently reported in this journal, that subclinical hypercortisolism was associated with overestimation of the glomerular filtration rate (eGFR) when calculated from serum creatinine (eGFRcre) in comparison to calculation from cystatin $\mathrm{C}$ (eGFRcys). The authors highlight the decrease in skeletal muscle mass as a possible attributing factor. Furthermore, they suggest that the dissociation between eGFRcre and eGFRcys could be used as a discriminating factor for subclinical hypercortisolism.

Indeed, calculation of eGFR using serum creatinine is limited due to the association with muscle mass which is reduced in various diseases, with increasing age and as the authors present also among patients with Cushing's syndrome and even subclinical hypercortisolism. Therefore, cystatin $\mathrm{C}$ has been suggested as a better marker for estimation of the GFR due to independence of muscle mass. However, Naka et al. only briefly discuss a possible effect of corticosteroids on cystatin $\mathrm{C}$ levels. We would like to therefore emphasize recent studies concerning this matter in greater detail.

Manetti et al. reported [2] a significant increase of cystatin C levels $(0.79 \mathrm{mg} / \mathrm{L}$ vs. $1.06 \mathrm{mg} / \mathrm{L})$ after intravenous application of methylprednisolone $(15 \mathrm{mg} / \mathrm{kg})$ among 23 patients with severe Graves' ophthalmopathy which

Ulf Schönermarck, Christina Dengler, Isaac Lean and Elisa Waldmann

Department of Medicine IV, University Hospital, LMU Munich, Marchioninistr. 15, D-81377 Munich, Germany Submitted May 28, 2020; Accepted Jun. 17, 2020 as EJ20-0321 Released online in J-STAGE as advance publication Jul. 9, 2020 Correspondence to: Ulf Schönermarck, MD, Department of Medicine IV, University Hospital, LMU Munich, Marchioninistr. 15, D-81377 Munich, Germany.

E-mail: ulf.schoenermarck@med.uni-muenchen.de was not seen among 44 control subjects $(0.83 \mathrm{mg} / \mathrm{L} v s$. $0.84 \mathrm{mg} / \mathrm{L})$. Similarly, prednisone treatment for symptomatic heart failure increased serum cystatin $\mathrm{C}$ concentration from $1.24 \mathrm{mg} / \mathrm{L}$ at baseline to 1.61 among 56 patients [3]. This effect may be dose dependent.

This clinical observation was recently supported in an animal model [4], where dexamethasone treatment increased plasma cystatin $\mathrm{C}$ levels in rats, without change in kidney function determined by inulin clearance. Further experiments demonstrated that the increased serum levels were a result of universally increased cystatin $\mathrm{C}$ production which was abolished by glucocorticoid-receptor mediated blockade. However, it is unknown if pharmacological (exogenous) glucocorticoids and endogenous cortisol exert similar effects on cystatin $\mathrm{C}$ metabolism nor if short and long term exposure have similar impact.

Cystatin $\mathrm{C}$ levels might be endogenously increased in several other conditions (e.g. hyperthyroidism) or diseases (e.g. in lung fibrosis). Accepting this hypothesis treatment of the underlying condition with corticosteroids would decrease elevated serum cystatin $\mathrm{C}$, as has been reported among patients with asthma [5].

Kidney function in patients with clinical or subclinical hypercortisolism should indeed be carefully interpreted as eGFRcre using serum creatinine might lead to an overestimation of the true GFR, while eGFRcys using cystatin $\mathrm{C}$ might underestimate this parameter. To elucidate the true impact of hypercortisolism on serum creatinine and cystatin $\mathrm{C}$ levels as well as GFR exact measurement of kidney function using inulin, DTPA or iohexol clearance or 24-hour creatinine clearance would be necessary.

\section{Conflict of Interest}

None of the authors have any potential conflicts of interest associated with this research.

\section{References}

1. Naka M, Kadoya M, Kosaka-Hamamoto K, Morimoto A, Miyoshi A, et al. (2020) Overestimation of glomerular filtration rate calculated from serum creatinine as compared with cystatin $\mathrm{C}$ in patients with subclinical hypercortisolism: Hyogo Adrenal Metabolic Registry. Endocr J 67: 469-476.

2. Manetti L, Genovesi M, Pardini E, Grasso L, Lupi I, et al. (2005) Early effects of methylprednisolone infusion on serum cystatin $\mathrm{C}$ in patients with severe Graves' 
ophthalmopathy. Clin Chim Acta 356: 227-228.

3. Zhai JL, Ge N, Zhen Y, Zhao Q, Liu C (2016) Corticosteroids significantly increase serum cystatin $\mathrm{C}$ concentration without affecting renal function in symptomatic heart failure. Clin Lab 62: 203-207.

4. Zhu XR, Ge N, Wang Y, Zhai JL, Liu C (2019) Corticosteroids significantly increase cystatin $\mathrm{C}$ levels in the plasma by promoting cystatin $\mathrm{C}$ production in rats. Ren Fail 41: 698-703.

5. Cimerman N, Brguljan PM, Krasovec M, Suskovic S, Kos J (2000) Serum cystatin C, a potent inhibitor of cysteine proteinases, is elevated in asthmatic patients. Clin Chim Acta 300: 83-95. 